\title{
Weapons of mass destruction and the role of the neurosurgeon
}

\author{
LEON E. MOORES, M.D. \\ Pediatric Neurosurgery, National Capital Consortium,Walter Reed Army Medical Center, \\ Washington, D.C.
}

\begin{abstract}
The desire to assist in a crisis should be tempered by a serious assessment of the technical preparation one has undertaken. Additionally, in the same way that a needs assessment is undertaken before prescribing a course of treatment, one should evaluate the actual staffing requirements of the situation. Many physician volunteers were turned away after the World Trade Center attacks because the overwhelming response of available medical personnel quickly exceeded the requirement. Finally, the duration and intensity of preparation should be based on a realistic evaluation of the likelihood of an event occurring that would necessitate use of the training. Before jumping into action in a situation in which weapons of mass destruction have been used, several issues must be addressed. Prior to the crisis, all professionals, neurosurgeons included, should ask how they can prepare themselves and their communities. During the crisis, neurosurgeons should evaluate their roles based on specialized triage training and experience, personal and equipment decontamination training, and the importance of neurosurgical skills. Finally, one should continually ask if there is anything he/she should be doing during the crisis in addition to performing neurosurgical tasks.
\end{abstract}

KEY WORDS - radiation • nuclear, biological, chemical warfare casualties - terrorism

In times of crisis it is a common human tendency to ask, "What can I do?" Certain groups of people are often even more willing to participate. There are countless stories of off-duty firemen and police officers who assisted during the September 11 attacks on the World Trade Center. Neurosurgeons are often somewhat aggressive, may be established leaders within their communities, and may even have a respected voice of authority. These qualities can contribute to the ability to assist effectively in a crisis. The desire to assist, however, should be tempered by a serious assessment of the technical preparation one has undertaken. In addition, similar to undertaking a needs assessment before prescribing a course of treatment, actual staffing requirements of the situation should be evaluated dispassionately. Many physician volunteers were turned away in New York because the overwhelming response of medical personnel quickly exceeded the demand. In other reports in this issue of Neurosurgical Focus the authors have covered specifics of treatment, protection, and triage. This final section will concentrate on several questions that are important to review prior to a WMD crisis.

Abbreviations used in this paper: US = United States; WMD = weapons of mass destruction.

\section{MASS CASUALTY TRAINING}

\section{Am I Able To Dispassionately Triage Patients in a Mass Casualty Situation?}

Typically, patients in the US are not turned away from emergency medical care because of a lack resources. In fact, a physician or hospital recommending this would be subject to professional and monetary penalties. In contrast, during a WMD event, there are several reasons why medical care may be withheld from individuals, potentially resulting in disability or death. Emergency medical systems and hospitals are likely to be overwhelmed. In this case, it would not be appropriate to treat the most ill patients first, contrary to common practice in US trauma centers. Severely injured patients require resource-intensive treatment plans. When those in a medical system encounter a patient requiring resources well beyond the existing capacity, the limited resources may be better used to treat three or four times as many less severely injured patients, ultimately saving more lives. The assumption here is diametrically opposed to our normal practicepatients with treatable injuries will die due to extraordinary demands on the system. The goal of mass-casualty triage is to minimize the total number of deaths. Addi- 
tionally, patients contaminated with chemical or biological products must be decontaminated prior to receiving care in a "clean" facility. Some patients may die during the procedure, but decontamination is essential to protect the integrity of the facility for all patients and caregivers. If this utilitarian model is unfamiliar, or if personal issues could prevent the neurosurgeon from being able to make the best decisions in these circumstances, he/she should likely refrain from engaging in emergency triage.

\section{WEAPONS OF MASS DESTRUCTION- SPECIFIC PERSONAL TRAINING}

\section{How Much do I Really Know About WMD Agents?}

Training in individual, equipment, and facility decontamination is available through local and regional chemical-biological teams, fire and rescue services, and military centers. Without specialized training, regular refresher training, and proper execution, neurosurgeons and their equipment may not be sufficiently decontaminated to participate safely in patient care. Reliance on local experts during the time of crisis and taking advantage of training sessions prior to any crisis are the best methods for ensuring the integrity of the system. Readiness, however, implies knowledge, not that chemical protective gear should be at the neurosurgeon's disposal at all times. According to the December 2001 issue of Scientific American, very few chemical experts own a protective mask. The difficulties associated with procurement and maintenance of the mask, the requirement of having it at one's disposal for use within seconds, and the minimal chance of encountering the chemical attack have led most to refrain from this protective measure.

\section{PERSONAL AND COMMUNITY ADVANCE PREPARATION}

\section{How Much Preparation Do We Need?}

During the preparatory phase, the credibility and leadership of neurosurgeons can be used to assist or guide the medical staff in making appropriate decisions for the benefit of the entire community. Significant resources in terms of personnel, time, and money will need to be committed to ensure an appropriate response is available in a crisis. Committee members may lose their focus, and strong leadership may be required to educate and/or energize the committee appropriately. During the preparatory phase, specific holding area and operating room procedures will be developed. It is important the neurosurgeons participate in these discussions to ensure that unnecessary restrictions are not placed on their ability to provide medical care. This must be balanced with the knowledge that the victim of a single improperly decontaminated chemical casualty may result in many more casualties among operating room personnel and other patients.

\section{ACTIONS DURING THE CRISIS}

\section{What Duties Should I Perform, in Addition to Neurosurgery, During the Crisis?}

With adequate preparation, every individual should be able to spot deficiencies in decontamination procedures and the handling of victims. Initial experiences with WMD training have demonstrated that it is common to fall back into old (noncontaminated) habits and forget the unique nature of medical management of these victims. It is therefore important for every individual to be alert for personal or system deficiencies. As a proven leader within his/her medical system, the neurosurgeon can be counted on to set the example. Not only should our personal procedures be up to date, but we should also ensure that other leaders within the system are evaluating themselves and their personnel.

\section{UNIQUE NEUROSURGICAL SKILLS}

\section{How Important Are My Skills?}

This is the last but perhaps most important question. As neurosurgeons, we provide a specialized but limited service in times of mass crisis. Additionally, from a utilitarian standpoint, there are very few people who can perform our roles. Should neurosurgical services be needed, the medical response system will depend on the neurosurgeon to provide those services. If the neurosurgeon is operating a triage center, he/she will not be available. A physician's assistant or experienced emergency room nurse can perform triage, but he/she cannot substitute for a neurosurgeon in the operating room. Unless the neurosurgeon is the only individual in the community who knows how to triage, decontaminate, and contact outside agencies for relief assistance, he/she should be performing neurosurgical tasks. If the neurosurgeon is the only individual who can perform all of these duties, he/she should consider training others before an attack occurs.

The strong desire to help must be placed within the context of a complex, coordinated emergency response system. Advance planning, training, and rehearsals can serve to solidify individual roles and identify system deficiencies. It is likely that neurosurgeons will best serve in any WMD attack by "standing by," ready to provide neurosurgical support.

Manuscript received January 19, 2002.

Accepted in final form February 20, 2002.

The opinions and views expressed in this article are those of the author and do not reflect the official policy or position of the US Army, Department of Defense, or US Government.

Address reprint requests to: Leon E. Moores, M.D., Walter Reed Army Medical Center, 6900 Georgia Avenue, Building 2, Room 6442, Washington D.C. 20307-5001. email: drleonmoores@ hotmail.com. 